\title{
Auch bei über 70-Jährigen effektiv und sicher
}

Hintergrund und Fragestellung: Die pulmonale Endarteriektomie (PEA) stellt die Therapie der Wahl bei Patienten mit chronischer thromboembolischer pulmonaler Hypertonie (CTEPH) dar. Allerdings gibt es nur wenige Literaturdaten über die Ergebnisse der PEA bei älteren Menschen. In dieser Studie wird der Frage nachgegangen, ob die Resultate und Risiken bei dieser Patientenpopulation vergleichbar mit denen jüngerer Patienten sind.

Patienten und Methoden: Insgesamt wurden 264 Patienten, die sich zwischen 2008 und 2012 einer PEA unterzogen, untersucht. Sie wurden nach ihrem Alter in zwei Gruppen eingeteilt: unter 70 - ( $n=176$, jüngere Patienten) und über 70 - Jährige $(n=88$, ältere Patienten).

Statistisch wurden Regressionsmodelle verwendet, um Vorhersagen zur perioperativen Mortalität und postoperativer Ereignisse zu identifizieren sowie deren Abhängigkeit vom Alter des Patienten.

\section{Originalie}

Vistarini N, Morsolini M, Klersy $C$ et al. Pulmonary endarterectomy in the elderly: safety, efficacy and risk factors.J Cardiovasc Med (Hagerstown). 2016 Feb;17(2):144-51.
Ergebnisse: Die perioperative Mortalität bei den älteren Patienten war insgesamt gering höher, aber nicht signifikant im Vergleich zu jüngeren Patienten (9,1 vs. 5,1\%, p $=0,22$ ). Es konnte eine Abhängigkeit vom Raucherstatus und einer präoperativen Sauerstofftherapie festgestellt

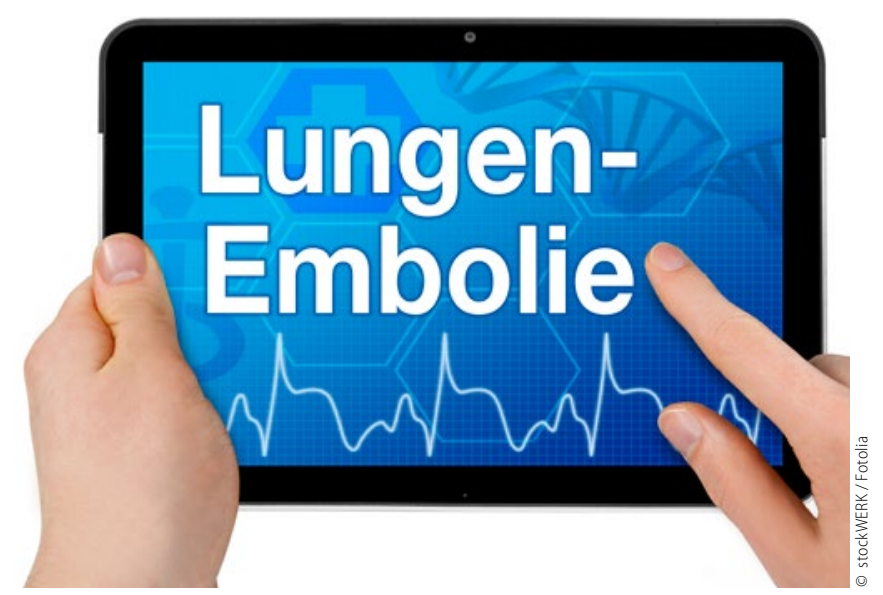

Bei chronischer thromboembolischer pulmonaler Hypertonie infolge rezidivierender Lungenembolien ist die pulmonale Endarteriektomie auch bei Älteren Mittel der Wahl.

werden. Die kumulative Überlebensrate nach 1, 2 und 4 Jahren betrug bei den jüngeren 93, 92 und $91 \%$ sowie 88,86 und $86 \%$ bei den älteren Patienten $(\mathrm{p}=0,19)$. Die klinische und hämodynamische Verbesserung war in beiden Gruppen ohne wesentlichen Unterschied.

Schlussfolgerung: Die PEA stellt auch bei älteren Patienten, trotz der etwas höheren perioperativen Mortalität, eine sichere und erfolgreiche Therapie der CTEPH dar.

\section{- Kommentar von Dr. med. Kathleen Gutjahr}

\section{Höheres Alter allein ist kein Grund, die Therapie vorzuenthalten}

Die PEA hat sich als Standarttherapie der CTEPH etabliert. Allein durch sie kann ein kurativer Ansatz verfolgt werden. Die Operation führt zu einer deutlichen Verbesserung der Hämodynamik, der funktionellen Klasse und der Prognose. Nach den aktuellen Empfehlungen der Weltkonferenz in Nizza müssen die Diagnostik und die Klärung der Operabilität durch ein erfahrenes CTEPH- Zentrum (30-50 PEA/Jahr mit einer perioperativen Letalität< $7 \%$ ) durchgeführt werden. In den großen Zentren liegt die perioperative Letalität bei $2-4 \%[1,2]$. Wie die vorliegende Studie zeigt, weicht diese bei Patienten über 70 Jahre nur wenig nach oben ab. Auch die Überlebensrate nach 1, 2 und 4 Jahren zeigt bei den älteren Patienten ähnlich gute Ergebnisse wie bei den jüngeren.

Durch die postoperative Verbesserung der Hämodynamik kommt es zu einer deutlichen und überwiegend anhaltenden Besserung der Lebensqualität der Patienten.

Die Studie zeigt eindrücklich, dass allein ein höheres Alter kein Grund sein darf, dem Patienten nach entsprechender Risikoabschätzung die einzig potenziell kurative Therapie der CTEPH vorzuenthalten.

\section{Literatur}

1. Jenkins D, Madani M, Mayer E et al:: Surgical treatment of chronic thromboembolic pulmonary hypertension. Eur.Respir J 41:735-742

2. Madani MM, Auger WR, Pretorius $V$ et al.: Pulmonary endarterectomy: recent changes in a single institutiones experience of more than 2700 patients. Am Thorac Surg 2012; 94:97-103; discussion

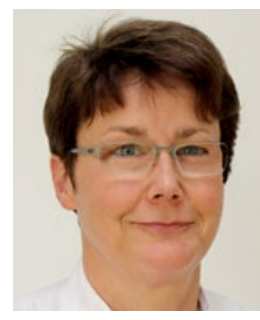

Dr. med. Kathleen Gutjahr

Robert-Koch-Klinikum

Thoraxzentrum des Klinikums St. Georg

Nikolai-Rumjanzew-Str. 100

04207 Leipzig

kathleen.gutjahr@sanktgeorg.de 